\title{
RANDOM APPROXIMATIONS AND RANDOM FIXED POINT THEOREMS FOR NON-SELF-MAPS
}

\author{
TZU-CHU LIN
}

(Communicated by John B. Conway)

\begin{abstract}
Recently, Sehgal and Singh [18] and Papageorgiou [16] considered different random versions of a very interesting theorem of Fan [4]. Instead of compact convex domain, here we consider a continuous condensing or nonexpansive random map defined on a closed ball (or closed convex set with bounded range). We prove it is true for certain spaces. As applications of our theorems, some random fixed point theorems of non-self-maps are derived.
\end{abstract}

1. Introduction and preliminaries. Random fixed point theory has just received much attention for the last decade, since the publication of the paper by Bharucha-Reid [1] in 1976, of which the stochastic version of the well-known Schauder's fixed point theorem was proved. In this paper, we will consider a stochastic version of a very interesting theorem of Fan [4, Theorem 2] which is stated as follows.

Let $K$ be a nonempty compact convex set in a normed linear space $X$. For any continuous map $f$ from $K$ into $X$, there exists a point $u$ in $K$ such that $\|u-f(u)\|=d(f(u), K)$.

Various aspects (nonstochastic) of this theorem has been studied by Fan [5], Ha [6], Lin [11-14], and Reich [17]. Recently, Sehgal and Singh [18], and Papageorgiou [16] gave stochastic versions of the above theorem. Here, instead of considering a continuous map defined on a compact convex set as in [18], we consider a continuous condensing or a nonexpansive map defined on a closed convex set with bounded range (or a closed ball). Roughly speaking in this paper, we will prove that the random version of above theorem is true for a continuous condensing random (for definitions see below) operator $f: \Omega \times S \rightarrow X$ where $S$ is a closed ball in a separable Banach space $X$ and $\Omega$ is a measurable space. We will also prove that the random version of above theorem is true for a continuous condensing (or nonexpansive) random operator $f: \Omega \times S \rightarrow X$ where $S$ is a closed convex subset of a separable Hilbert space and $f(\omega, S)$ is bounded for $\omega \in \Omega$. As applications of our theorems, some stochastic fixed point theorems are derived. We also remark that a related (but different) result has been obtained by Sehgal and Waters [19].

Now, we state some definitions.

Let $(\Omega, \Sigma)$ be a measurable space with $\Sigma$ a sigma algebra of subsets of $\Omega$. Let $X$ be a Banach space, a map $f: \Omega \rightarrow X$ is called measurable if for each open set $B$ of

Received by the editors January 15, 1987 and, in revised form, July 6, 1987. This paper was presented at the 841th meeting of the American Mathematical Society held at the University of Tennessee at Knoxville, Tennessee, March 25-26, 1988.

1980 Mathematics Subject Classification (1985 Revision). Primary 47H10, 60H25; Secondary $41 \mathrm{~A} 50$. 
$X, f^{-1}(B) \in \Sigma$. If $f$ is a multifunction, then $f^{-1}(B)=\{\omega \mid f(\omega) \cap B \neq \varnothing\}$. Let $S$ be a nonempty subset of $X$, and a map $f: \Omega \times S \rightarrow X$ is called a random operator if for each fixed $x \in S$, the map $f(\cdot, x): \Omega \rightarrow X$ is measurable. A measurable map $\varphi: \Omega \rightarrow S$ is a random fixed point of the random operator $f$ if $f(\omega, \varphi(\omega))=\varphi(\omega)$, for each $\omega \in \Omega$.

Let $B$ be a nonempty bounded subset of $S$, and let $\alpha(B)=\inf \{c>0 \mid B$ can be covered by a finite number of sets of diameter $\leq c\}$. A map $f: S \rightarrow X$ is called condensing (e.g. see [15]) if for each bounded subset $B$ of $S$ with $\alpha(B)>0$, we have $\alpha(f(B))<\alpha(B) ; f$ is nonexpansive if $\|f(x)-f(y)\| \leq\|x-y\|$ for $x, y \in S$; $f$ is compact if $f(S)$ is totally bounded.

It is easy to see (e.g. see [15]) that (i) $\alpha(A) \leq \alpha(B)$ if $A \subset B$, (ii) $\alpha(\operatorname{co} A)=\alpha(A)$, (iii) $\alpha(A+B) \leq \alpha(A)+\alpha(B)$, (iv) $\alpha(A)=0$ iff $A$ is totally bounded.

A random operator $f: \Omega \times S \rightarrow X$ is continuous (condensing, nonexpansive, compact etc.) if for each $\omega \in \Omega, f(\omega, \cdot)$ is continuous (condensing, nonexpansive, compact etc.) (e.g. see Itoh [8]); $f$ is a contraction if there exists a map $k: \Omega \rightarrow[0,1)$ satisfying $\|f(\omega, x)-f(\omega, y)\| \leq k(\omega)\|x-y\|$ for $x, y \in S, \omega \in \Omega$ (see [19]).

\section{Main results.}

THEOREM 1. Let $S$ be a closed ball with center at origin and radius $r$ in a separable Banach space $X$, and $f: \Omega \times S \rightarrow X$ be a continuous condensing random operator. Then there exists a measurable map $\varphi: \Omega \rightarrow S$ such that

$$
\|\varphi(\omega)-f(\omega, \varphi(\omega))\|=d(f(\omega, \varphi(\omega)), S)
$$

for each $\omega \in \Omega$.

ProOF. Define $R: X \rightarrow S$ by

$$
R(x)= \begin{cases}x & \text { if }\|x\| \leq r \\ \frac{r x}{\|x\|} & \text { if }\|x\| \geq r .\end{cases}
$$

From Nussbaum [15, Corollary 1], $R$ is a continuous 1-set-contractive map. Define $F: \Omega \times S \rightarrow S$ by $F(\omega, x)=R(f(\omega, x))$. It is easy to see that $F$ is a continuous condensing random operator. From Itoh [8, Theorem 2.1], there exists a random fixed point $\varphi: \Omega \rightarrow S$ of $F$, i.e. $F(\omega, \varphi(\omega))=\varphi(\omega)$ for each $\omega \in \Omega$. Now, we will prove that this measurable map $\varphi$ satisfies the desired property. For each $\omega \in \Omega$,

$$
\begin{aligned}
& \|\varphi(\omega)-f(\omega, \varphi(\omega))\|=\|F(\omega, \varphi(\omega))-f(\omega, \varphi(\omega))\| \\
& =\|R(f(\omega, \varphi(\omega)))-f(\omega, \varphi(\omega))\| \\
& =\left\{\begin{array}{rr}
\|f(\omega, \varphi(\omega))-f(\omega, \varphi(\omega))\|=0, & \text { if }\|f(\omega, \varphi(\omega))\| \leq r, \\
\left\|\frac{r f(\omega, \varphi(\omega))}{\|f(\omega, \varphi(\omega))\|}-f(\omega, \varphi(\omega))\right\|= & \|f(\omega, \varphi(\omega))\|-r, \\
\text { if }\|f(\omega, \varphi(\omega))\| \geq r .
\end{array}\right.
\end{aligned}
$$

For any $y \in S$, we have

$$
\|f(\omega, \varphi(\omega))\|-r \leq\|f(\omega, \varphi(\omega))\|-\|y\| \leq\|f(\omega, \varphi(\omega))-y\| .
$$

Therefore

$$
\|\varphi(\omega)-f(\omega, \varphi(\omega))\|=d(f(\omega, \varphi(\omega)), S)
$$


THEOREM 2. Let $S$ be a nonempty separable closed convex subset of a Hilbert space $X$, and $f: \Omega \times S \rightarrow X$ be a continuous condensing random operator such that $f(\omega, S)$ is bounded, for any $\omega \in \Omega$.

Then there exists a measurable map $\varphi: \Omega \rightarrow S$ such that

$$
\|\varphi(\omega)-f(\omega, \varphi(\omega))\|=d(f(\omega, \varphi(\omega)), S),
$$

for each $\omega \in \Omega$.

PROOF. Let $p$ be the proximity map on $S$, i.e. $p$ is a continuous map from $X$ into $S$ such that for each $y \in X$ we have

$$
\|p(y)-y\|=d(y, S) .
$$

From [3], $p$ is nonexpansive in Hilbert space. Then $p \circ f: \Omega \times S \rightarrow S$. It is easy to see that $p \circ f$ is a continuous condensing random operator and $p \circ f(\omega, S)$ is bounded, for any $\omega \in \Omega$. From Itoh [8, Theorem 2.1], there exists a random fixed point of $p \circ f$, i.e. there exists a measurable $\operatorname{map} \varphi: \Omega \rightarrow S$ such that $p \circ f(\omega, \varphi(\omega))=\varphi(\omega)$, for each $\omega \in \Omega$. Hence

$$
\begin{aligned}
\|\varphi(\omega)-f(\omega, \varphi(\omega))\| & =\|p \circ f(\omega, \varphi(\omega))-f(\omega, \varphi(\omega))\| \\
& =d(f(\omega, \varphi(\omega)), S), \quad \text { for each } \omega \in \Omega .
\end{aligned}
$$

COROllary 1 (Sehgal AND Waters [19, Theorem 3]). Let $S$ be a nonempty separable closed convex subset of a Hilbert space $X$ and $g, h: \Omega \times S \rightarrow X$ be random operators such that $g$ is a contraction and $h$ is compact and continuous.

Then there exists a measurable map $\varphi: \Omega \rightarrow S$ such that

$$
\|\varphi(\omega)-f(\omega, \varphi(\omega))\|=d(f(\omega, \varphi(\omega)), S),
$$

where $f=g+h$. If additionally $f(\Omega \times \partial S) \subseteq S$, then $\varphi$ is a random fixed point.

ProOF. For each fixed $\omega \in \Omega$, and each bounded subset $A$ of $S$, with $\alpha(A)>0$, we have

$$
\begin{aligned}
\alpha(f(\omega, A)) & \leq \alpha(g(\omega, A)+h(\omega, A)) \leq \alpha(g(\omega, A))+\alpha(h(\omega, A))=\alpha(g(\omega, A)) \\
& \leq k(\omega) \alpha(A)<\alpha(A) .
\end{aligned}
$$

So $\dot{f}$ is condensing. Hence this corollary follows immediately from Theorem 2 and the Remark after Theorem 4.

THEOREM 3. Let $S$ be a nonempty closed convex bounded subset of a separable Hilbert space $X$, and $f: \Omega \times S \rightarrow X$ be a nonexpansive random operator. Then there exists a measurable map $\varphi: \Omega \rightarrow S$ such that

$$
\|\varphi(\omega)-f(\omega, \varphi(\omega))\|=d(f(\omega, \varphi(\omega)), S),
$$

for each $\omega \in S$.

PROOF. Let $p$ be the same as in the proof of Theorem 2. Then $p \circ f: \Omega \times S \rightarrow S$ is also a nonexpansive random operator. From Itoh [8, Theorem 2.5], there exists a measurable map $\varphi: \Omega \rightarrow S$ such that $p \circ f(\omega, \varphi(\omega))=\varphi(\omega)$, for each $\omega \in \Omega$. Hence

$$
\|\varphi(\omega)-f(\omega, \varphi(\omega))\|=d(f(\omega, \varphi(\omega)), S),
$$

for each $\omega \in \Omega$. 
We remark that the reason we can have weaker assumption for Theorem 2, namely $f(\omega, S)$ is bounded for $\omega \in \Omega$ (instead of $S$ is bounded in Theorem 3) and $S$ is separable (instead of $X$ is separable in Theorem 3 ) is that corresponding random fixed point theorem for condensing random self-maps is available (see Itoh [8]). We will consider a weaker assumption for nonexpansive maps after Theorem 6. Certainly, Theorem 1 is still true, if we assume that $S$ is separable (instead of $X$ is separable). (The proof is exactly the same as Theorem 1.) The same remark can be made for the following Theorem 4 .

THEOREM 4. Let $S$ be a closed ball with center at origin and radius $r$ in a separable Banach space $X$, and $f: \Omega \times S \rightarrow X$ be a continuous condensing random operator. Moreover, $f$ satisfies one of the following conditions:

(i) For each $\omega \in \Omega$, each $x \in S$ with $x \neq f(\omega, x)$, there exists $y$, depending on $\omega$ and $x$, in $I_{S}(x)=\{x+c(z-x) \mid$ some $z \in S, c>0\}$ such that $\|y-f(\omega, x)\|<$ $\|x-f(\omega, x)\|$.

(ii) $f$ is weakly inward (i.e. for each $\omega \in \Omega, f(\omega, x) \in \overline{I_{S}(x)}$ for $x \in S$ ). Then $f$ has a random fixed point.

PROOF. Let $f$ satisfy condition (i). From Theorem 1 , there exists a measurable $\operatorname{map} \varphi: \Omega \rightarrow S$ such that

$$
\|\varphi(\omega)-f(\omega, \varphi(\omega))\|=d(f(\omega, \varphi(\omega)), S)
$$

for each $\omega \in \Omega$.

We will prove that $\varphi$ is the desired random fixed point. If there exists $\omega \in \Omega$ such that $\varphi(\omega) \neq f(\omega, \varphi(\omega))$. From the assumption (i), there exists $y$ in $I_{S}(\varphi(\omega))$, such that

$$
\|y-f(\omega, \varphi(\omega))\|<\|\varphi(\omega)-f(\omega, \varphi(\omega))\| .
$$

Since $y \in I_{S}(\varphi(\omega))$, there exists $z \in S, c>0$ such that $y=\varphi(\omega)+c(z-\varphi(\omega))$. Since $y \notin S$, otherwise contradict the choice of $\varphi$, we can assume that $c>1$. Then $z=y / c+(1-1 / c) \varphi(\omega)=(1-\beta) y+\beta \varphi(\omega)$, where $\beta=1-1 / c, 0<\beta<1$. Therefore

$$
\begin{aligned}
\|z-f(\omega, \varphi(\omega))\| & \leq(1-\beta)\|y-f(\omega, \varphi(\omega))\|+\beta\|\varphi(\omega)-f(\omega, \varphi(\omega))\| \\
& <(1-\beta)\|\varphi(\omega)-f(\omega, \varphi(\omega))\|+\beta\|\varphi(\omega)-f(\omega, \varphi(\omega))\| \\
& =\|\varphi(\omega)-f(\omega, \varphi(\omega))\|
\end{aligned}
$$

which contradict the choice of $\varphi$. Hence $f(\omega, \varphi(\omega))=\varphi(\omega)$ for each $\omega \in \Omega$, and $\varphi$ is the desired random fixed point of $f$.

If $f$ satisfies condition (ii). For each $\omega \in \Omega$, each $x \in S$ with $x \neq f(\omega, x)$, since $f(\omega, x) \in \overline{I_{S}(x)}$, there exists $y$ in $I_{S}(x)$ such that $\|y-f(\omega, x)\|<\|x-f(\omega, x)\|$, and $f$ satisfies condition (i).

REMARK. If $f(\omega, \partial S) \subset S$ for each $\omega \in \Omega$, then $f$ satisfies condition (ii) of Theorem 4; because $S \subset I_{S}(x)$, and $I_{S}(x)=X$ if $x \in \operatorname{int} S$, where $\partial S$, int $S$ denote the boundary and interior of $S$, respectively.

THEOREM 5. Let $S$ be a nonempty separable closed convex subset of a Hilbert space $X$, and $f: \Omega \times S \rightarrow X$ be a continuous condensing random operator such that $f(\omega, S)$ is bounded for any $\omega \in \Omega$. Moreover, $f$ satisfies any one of the conditions (i), (ii) in Theorem 4. Then $f$ has a random fixed point. 
Proof. The proof is similar to the proof of Theorem 4. Instead of using Theorem 1, we use Theorem 2 .

Similarly, we have the following Theorem 6.

THEOREM 6. Let $S$ be a nonempty closed convex bounded subset of a separable Hilbert space $X$, and $f: \Omega \times S \rightarrow X$ be a nonexpansive random operator. Moreover $f$ satisfies any one of the conditions (i), (ii) in Theorem 4. Then $f$ has a random fixed point.

For nonexpansive maps, whether we can only assume that $f(\omega, S)$ is bounded for $\omega \in \Omega$ instead of the stronger condition that $S$ is bounded? We will prove that it is true, under the restriction, namely $\Sigma$ is closed under Suslin operation (for definition of this, see e.g. [20]). We note that there are several different definitions for the measurability of a function $f: \Omega \rightarrow X$ in the literature (see $[\mathbf{2}, \mathbf{7}, \mathbf{2 0}]$ ); for example, some author called $f$ is measurable if $f^{-1}(B) \in \Sigma$ for every closed subset $B$ of $X$. However, these different definitions are all equivalent, if $\Sigma$ is closed under Suslin operation and $f$ is closed valued (e.g. see [20, Theorem 4.2]).

For the following Lemma 1 , Theorems $3^{\prime}, 6^{\prime}$, we need assume that $\Sigma$ is closed under the Suslin operation.

LEMMA 1. Let $S$ be a nonempty closed convex subset of a separable uniformly convex Banach space $X$, and $f: \Omega \times S \rightarrow S$ be a nonexpansive random operator such that $f(\omega, S)$ is bounded, for any $\omega \in \Omega$. Then $f$ has a random fixed point.

Proof. Let $C(\omega)=\overline{\mathrm{co}}(f(\omega, S))$, for $\omega \in \Omega$. Let $D=\left\{x_{n} \mid n \geq 1\right\}$ be a countable dense subset of $S$, then it is easy to check that

$$
C(\omega)=\overline{\mathrm{co}} \bigcup_{n \geq 1} f\left(\omega, x_{n}\right)
$$

From [7, Proposition 2.3 and Theorem 9.1], $C: \Omega \rightarrow S$ is a measurable multifunction. Consider the multifunction $L(\cdot)$ defined by $L(\omega)=\{x \in C(\omega) \mid f(\omega, x)=x\}$. For each $\omega \in \Omega, C(\omega)$ is a closed convex bounded subset of $S$ and $f(\omega, \cdot): C(\omega) \rightarrow$ $C(\omega)$, from Kirk [9], $f(\omega, \cdot)$ has a (deterministic) fixed point in $C(\omega)$, i.e. $L(\omega) \neq \varnothing$. It is clear that $L(\cdot)$ is closed valued and $\operatorname{Gr}(L)=\{(\omega, x) \in \Omega \times S \mid f(\omega, x)-x=$ $0\} \cap \operatorname{Gr}(C)$, where $\operatorname{Gr}(C)=\{(\omega, x) \in \Omega \times S \mid x \in C(\omega)\}$.

As we pointed out in the paragraph after Theorem 6 , for a closed valued map, all different definition of measurability in the literature are equivalent, if $\Sigma$ is closed under the Susin operation. So we will not distinguish them for the rest of our proof. Since $(\omega, x) \rightarrow f(\omega, x)-x$ is a Carathéodory function (see e.g. [20, p. 863]), from [7, Theorem 6.1] (or see [2, p. 70, Lemma III. 14]), this map is jointly measurable. Since $C(\cdot)$ is measurable, from [20, Theorem 4.2(d)], $\operatorname{Gr}(C) \in \Sigma \times B(X)$, where $B(X)=$ Borel $\sigma$-field of $X$. Thus $\operatorname{Gr}(L) \in \Sigma \times B(X)$. Since $L(\cdot)$ is closed valued, from [20, Theorem $4.2(\mathrm{~g})], L(\cdot)$ is measurable. From the Kuratowski-Ryll Nardzewski selection theorem [10], there exists a measurable function $\varphi: \Omega \rightarrow S$ such that $\varphi(\omega) \in L(\omega)$, for each $\omega \in \Omega$. Therefore $\varphi$ is a random fixed point of $f$.

The proof of Lemma 1 is essentially due to the referee, who suggests these arguments to prove the following Theorem $3^{\prime}$. The author modifies his argument to derive and find our Lemma 1 . We note that Itoh $[8$, Theorem 2.5] proved the above Lemma is true for a weakly compact convex subset $S$, without assuming that $\Sigma$ is 
closed under Suslin operation. The above lemma is still true if we assume that $X$ is a separable Banach space with normal structure (for definition of this, see Kirk [9]). The proof of this is exactly the same as Lemma 1 .

THEOREM $3^{\prime}$. Let $S$ be a nonempty closed convex subset of a separable Hilbert space $X$, and $f: \Omega \times S \rightarrow X$ be a nonexpansive random operator such that $f(\omega, S)$ is bounded, for any $\omega \in \Omega$. Then there exists a measurable map $\varphi: \Omega \rightarrow S$ such that

$$
\|\varphi(\omega)-f(\omega, \varphi(\omega))\|=d(f(\omega, \varphi(\omega)), S) .
$$

PROOF. The proof is exactly the same as Theorem 3 , instead of using Itoh [8, Theorem 2.5], we use Lemma 1 to show that $p \circ f$ has a random fixed point.

THEOREM $6^{\prime}$. Let $S$ be a nonempty closed convex subset of a separable Hilbert space $X$, and $f: \Omega \times S \rightarrow X$ be a nonexpansive random operator such that $f(\omega, S)$ is bounded for any $\omega \in \Omega$. Moreover, $f$ satisfies any one of the conditions (i), (ii) in Theorem 4. Then $f$ has a random fixed point.

ProOF. Similar to Theorem 6.

Therefore, for the case that $\Sigma$ is closed under Suslin operation, we have more general Theorem $3^{\prime}, 6^{\prime}$ (instead of their special case Theorem 3 and 6 respectively).

ACKNOWLEDGEMENT. I would like to thank the referee for telling me about the paper by Sehgal and Waters [19] and for the helpful suggestions and comments.

\section{REFERENCES}

1. A. T. Bharucha-Reid, Fixed point theorems in probabilistic analysis, Bull. Amer. Math. Soc. 82 (1976), 641-645.

2. C. Castaing and M. Valadier, Convex analysis and measurable multifunctions, Lecture Notes in Math., vol. 580, Springer-Verlag, Berlin and New York, 1977.

3. W. Cheney and A. A. Goldstein, Proximity maps for convex sets, Proc. Amer. Math. Soc. 10 (1959), 448-450.

4. Ky Fan, Extensions of two fixed point theorems of F. E. Browder, Math. Z. 112 (1969), 234-240.

5. __ Some properties of convex sets related to fixed point theorems, Math. Ann. 266 (1984), $519-537$.

6. C. W. Ha, Extensions of two fixed point theorems of Ky Fan, Math. Z. 190 (1985), 13-16.

7. C. J. Himmelberg, Measurable relations, Fund. Math. 87 (1975), 53-72.

8. S. Itoh, Random fixed point theorems with an application to random differential equations in Banach spaces, J. Math. Anal. Appl. 67 (1979), 261-273.

9. W. A. Kirk, A fixed point theorem for mappings which do not increase distances, Amer. Math. Monthly 72 (1965), 1004-1006.

10. K. Kuratowski and C. Ryll-Nardzewski, A general theorem on selector, Bull. Acad. Polon. Sci. Ser. Sci. Math. Astronom. Phys. 13 (1965), 397-403.

11. T. C. Lin, A note on a theorem of Ky Fan, Canada Math. Bull. 22 (1979), 513-515.

12. , Convex sets, fixed points, variational and minimax inequalities, Bull. Austral. Math. Soc. 34 (1986), 107-117.

13. _ Approximation theorems and fixed point theorems in cones, Proc. Amer. Math. Soc. 102 (1988), 502-506.

14. T. C. Lin and C. L. Yen, Applications of the proximity map to fixed point theorems in Hilbert space, J. Approximation Theory 52 (1988), 141-148.

15. R. D. Nussbaum, The fixed point index for local condensing maps, Ann. Mat. Pura Appl. 89 (1971), 217-258.

16. N. S. Papageorgiou, Random fixed point theorems for measurable multifunctions in Banach spaces, Proc. Amer. Math. Soc. 97 (1986), 507-514. 
17. S. Reich, Approximate selection, best approximations, fixed points and invariant sets, J. Math. Anal. Appl. 62 (1978), 104-112.

18. V. M. Sehgal and S. P. Singh, On random approximations and a random fixed point theorem for set valued mappings, Proc. Amer. Math. Soc. 95 (1985), 91-94.

19. V. M. Sehgal and C. Waters, Some random fixed point theorems for condensing operators, Proc. Amer. Math. Soc. 90 (1984), 425-429.

20. D. H. Wagner, Survey of measurable selection theorems, SIAM J. Control Optim. 15 (1977), 859-903.

DEPARTMENT OF MAThEMATICS, UNIVERSity OF Wisconsin-MilWAUKEe, MilWAUKEE, WISCONSIN 53201 